\title{
Home management of malaria in an academic community - University of Benin, Benin City, Nigeria
}

\author{
VA Wagbatsoma ${ }^{a}$, JE Obomighie and NH Nwokike $^{a}$
}

\begin{abstract}
Malaria remains one of the world's most devastating diseases, killing millions of people yearly. Home management is an important strategy adopted for the reduction of its fatality. This survey to evaluate home management practices towards malaria among heads of households was undertaken at the residential quarters of the University of Benin, Benin City, Nigeria. The residential quarters consists of 239 housing units; 179 units accommodating senior workers and 60 units accommodating junior workers. Using the systematic sampling method, a sampling ratio of 1:2 was used in selecting a total of 120 housing units and 120 heads of households for the study. A structured intervieweradministered questionnaire was used for data collection. Results show that of the 120 heads of households interviewed $70.8 \%$ were males, while $29.2 \%$ were females. Knowledge of the cause of malaria was poor 46 (38.3\%). However, knowledge improved with increase in level of education and this association was found to be statistically significant $(\mathrm{p}=0.002)$. The common signs and symptoms associated with malaria in descending order of prevalence were fever $83.3 \%$, weakness $70.0 \%$, headache $62.5 \%$, body pains $50.8 \%$, vomiting $47.7 \%$ and loss of appetite $40.8 \%$. Majority of respondents (52.5\%) delayed treatment to between one to three days after recognition of signs/ symptoms of malaria. Most of the respondents (90.8\%) preferred orthodox drugs for home treatment of malaria while 3.3\% and 5.8\% used herbal preparations. Others used a combination of orthodox and herbal preparations. The commonly used orthodox drugs were choloroquine $(45.0 \%)$, followed by sulphadoxine pyrimethamine (fansidar) $(25.0 \%)$ and sulphadaxin pyrimethamine (maloxine) (20.0\%), amongst others. However, chloroquine, which was most commonly used, was the most incorrectly administered. Prevention against malaria was mostly achieved through the use of door/window gauze (35\%) and combination of insecticide and door/window gauze (30\%). Home management of malaria is an important strategy that needs to be sustained. However, inadequate treatment regimen remains a hindrance to the success of this vital control effort. There is a need to educate all strata of the society on the correct administration of common antimalarials to reduce the impact of the disease.
\end{abstract}

Key WorDS: Malaria, home management, University of Benin, treatment

${ }^{a}$ Department of Community Health, School of Medicine, College of Medical Sciences, University of Benin, Benin City, Nigeria.

Correspondence: VA Wagbatsoma, Department of Community Health, School of Medicine, College of Medical Sciences, University of Benin, Benin City, Nigeria.

(C) CMS UNIBEN JMBR 2004; 3(1): 73-80

\section{INTRODUCTION}

Malaria is one of the world's most devastating diseases. Nearly 500 million people suffer from acute malaria each year, the majority of which are children. ${ }^{1}$ About one million people worldwide die yearly from the disease; about 3,000 daily, of which $90 \%$ are from sub- 
Saharan Africa. ${ }^{1,2}$ Africa is worst hit due to a combination of factors, which include weak health care systems, large population movement, deteriorating sanitation, climatic changes, increasing drug resistance and uncontrolled developmental activities. ${ }^{1,3}$ The WHO Roll Back Malaria project, which supports the Global Roll Back Malaria partnership, aims to coordinate global action to fight malaria. The tools for malaria control that will be implemented through the initiative include insecticide-treated bed nets, rectal suppositories, package of essential interventions for care of the sick child and simple packaging of antimalarials to help ensure that people take the proper course of treatment. Other tools are improved referral for severe malaria by mother and traditional healers, and the training of drug suppliers in the provision and counselling for safe use of antimalarials. ${ }^{4}$ In the rural areas of Africa, including Nigeria, more than two third of malaria episodes are self-treated, while about half are self-treated in urban areas. ${ }^{5}$ Most (96\%) of the treatments, according to Umeh, ${ }^{5}$ are promptly administered within 24 hours. Unfortunately, only about $14.3 \%$ correctly administer the antimalarial drugs. ${ }^{5}$

The nearer the treatment venue is to the home the more likely the treatment will start early. The earlier the treatment starts the better for reduction of complications and eventual death. This all important malaria control strategy lies in the hands of lay people (mothers and care givers) in the community who would usually commence treatment at home.

This survey was undertaken at the University of Benin staff quarters to assess the management, at the household level, of malaria among residents in an academic community, where the level of education is high and generally better than that of the larger community. We hope that this will give an insight into the appropriateness and usefulness of this practice.

\section{MATERIALS AND METHODS}

\section{Study area}

The study was conducted at the staff quarters of the University of Benin, Benin City, Nigeria. The campus is located along the Benin-Lagos expressway. The staff quarters consists of 239 housing units, of which 179 (75\%) are for senior staff and are categorised into P, A, B and $\mathrm{D}$, made up of $15,48,80$ and 36 units respectively. The remaining 60 housing units are for junior staff.

\section{Survey method}

Houses on campus are linearly built on both sides of tarred motorable roads. Stratified systematic sampling method was employed to determine the households used for the survey, using a sampling ratio of 1:2. A total of 120 houses were selected for the survey from the various categories of housing units. Heads of households of the selected houses were consequently selected for the survey. An interviewer-administered structured questionnaire was used for data collection after obtaining necessary informed consent. The interviewers were 600 level medical students who were specially trained for the administration of the questionnaire. The questionnaire sought information on causes of malaria, diagnostic features for malaria, drug of choice for treatment and treatment regimen, and common preventive measures adopted in the home. Questions asked about knowledge included what causes malaria and how it is transmitted. Knowledge of the cause of malaria was taken as correct if Plasmodium or malaria parasite was mentioned, and incorrect if any other thing was stated. Mode of transmission was accepted as correct if mosquito was proffered.

Data were analysed using PEPI, the computer programme for epidemiological studies. Association was established using chi-square test for statistical significance. 


\section{RESULTS}

Of the 120 heads of households surveyed, 85 (70.8\%) were males while $35(29.2 \%)$ were females. Their ages ranged from 31 to 65 years with a mean age of $49.1 \pm 6.9$ years. A majority of the respondents (85.0\%) were within ages 41 to 60 years.

Knowledge of the cause of malaria was generally poor. Forty six (38.3\%) had correct knowledge while 74 (61.7\%) had incorrect knowledge of the cause of malaria. Incorrect causes of malaria proffered included hard work, long exposure to sunlight, bacteria and flies, among others. Knowledge improved with increase in the level of education, and this association was found to be statistically significant $(\mathrm{p}=0.002)$ (Table 1$)$.
The common recognisable signs/symptoms reported in order of prevalence were fever (83.3\%), weakness (70.0\%), headache (62.5\%), body pains (60.8\%), vomiting (46.7\%) and loss of appetite (40.8\%) (Figure 1). In spite of the early recognition of symptoms, treatment was delayed in most cases. Sixty three $(52.5 \%)$ commenced treatment between one and three days.

Majority (90.8\%) of the study population preferred treating malaria with orthodox medicines. The choice of orthodox medication for the treatment of malaria increased as level of education increased, although this association was not statistically significant ( $\mathrm{p}$ $=0.80)$. Surprisingly, the use of herbal medication and combination of orthodox and

Table 1 Influence of level of education on knowledge of the cause of malaria

\begin{tabular}{lrrrr}
\hline Level of education & Correct knowledge & Incorrect knowledge & \multicolumn{2}{r}{ Total } \\
\hline Primary & 0 & 7 & $(5.8)$ & $7(5.8)$ \\
Secondary & $1(0.8)$ & $16(13.3$ & $17(14.2)$ \\
Tertiary & $45(37.5)$ & $51(42.5)$ & $96(80.0)$ \\
\hline Total & $46(38.3)$ & $74(61.7)$ & $120(100)$ \\
\hline
\end{tabular}

$\chi^{2}=14.9 ; d f=2 ; p=0.002$

\section{Figure 1 Prevalence of common signs and symptoms}


herbal preparations were observed among those with tertiary education (Table 2).

Table 3 shows the commonly used orthodox drugs in the home. One hundred and twenty five (79.1\%) administered antimalarials correctly, while 33 (20.9\%) incorrectly treated malaria and the difference was statistically significant $(p=0.002)$. The most adequately administered drug in the home treatment of malaria was sulphadoxine pyrimethamine (maloxine) (95.8\%), followed by chloroquine $(81.5 \%)$ and the antipyretic paracetamol $(80.0 \%)$, while halofantrine (halfan) was the most incorrectly used antimalarial drug.

Various reasons were given for the choice of medicines used for home treatment of malaria (Figure 2). Most of the respondents (32.5\%) claimed that orthodox drugs are more effective, some (17.5\%) said it is quantifiable and safer, yet others (14.2\%) claimed that it is a product of scientific research. Some used it because they have good knowledge of the drugs. Of those who used herbal preparations, $29.2 \%$ claimed that they were effective, while others (5.8\%) used it because they felt most orthodox drugs are fake. However, 3.3\% claimed that they used herbal preparation when orthodox drugs failed and because herbal concoctions were readily available.

The respondents used different malaria prevention methods. The commonest method was the use of wire mesh on door/window (29.2\%), while $25.0 \%$ used door/window mesh alongside insecticides. Some others combined sleeping under untreated bed nets with keeping their surroundings clean. However, 2.5\% prevented malaria by sleeping under untreated bed nets, spraying insecticide and prophylaxis treatment.

Table 2 Preferred mode of malaria treatment by level of education

\begin{tabular}{lrclr}
\hline Level of education & Orthodox & Orthodox + herbal & Herbal & Total \\
\hline Primary & $7(5.8)$ & 0 & 0 & $7(5.8)$ \\
Secondary & $16(13.3)$ & $1(0.8)$ & 0 & $17(14.2)$ \\
Tertiary & $86(71.7)$ & $6(5.0)$ & $4(3.3)$ & $96(80.0)$ \\
\hline Total & $109(90.8)$ & $7(5.8)$ & $4(3.3)$ & $120 \quad(100)$ \\
\hline$\chi^{2}=1.53 ; d f=4 ; p=0.80$ & & &
\end{tabular}

Table 3 Frequency of the correct dosage of commonly used orthodox drugs

\begin{tabular}{lrrrr}
\hline Commonly used drugs & Correct dosage & Incorrect dosage & Total \\
\hline Chloroquine & $44(27.8)$ & $10(6.3)$ & $54(34.2)$ \\
Sulphadoxine pyrimethamine (fansidar) & $23(14.6)$ & 7 & $(4.4)$ & $30 .(19.0)$ \\
Sulphadoxine pyrimethamine (maloxine) & $23(14.6)$ & 1 & $(0.6)$ & $24(15.2)$ \\
Chloroquine sulphate (nivaquine) & $15(9.5)$ & 4 & $(2.5)$ & $19(12.0)$ \\
Halofantrine (halfan) & $3(1.9)$ & 7 & $(4.4)$ & $10(6.3)$ \\
Paracetamol & $17(10.8)$ & 4 & $(2.5)$ & $21(13.3)$ \\
\hline Total & $125(79.1)$ & $33(20.9)$ & $158(100)$ \\
\hline
\end{tabular}

$\chi^{2}=18.85 ; d f=5 ; p=0.002$ 
Figure 2 Reasons for using orthodox medicine

Figure 3 Reasons for using herbal medication

\section{DISCUSSION}

Males, as expected, dominated the household heads, and only a few females (29.2\%) were heads of households. This observation is consistent with the report of the Nigerian National Population Demographic Survey. ${ }^{6}$ The current circular letter from the Federal Ministry of Education fixed the retirement age for university staff between 60 and 65 years. $^{7}$ The age range for the study population of 31 to 65 years and mean age of $49.1 \pm 6.9$ years falls within the description.

The poor knowledge of the cause of malaria has been previously documented ${ }^{8}$ as observed in this survey. However, the poor knowledge of the cause of malaria among the 
population is surprising. Contrary to opinions, a minimum of secondary education improved knowledge on health matters. ${ }^{9}$ The low level of knowledge about the cause of malaria in a citadel of learning of this nature is an indication that outside the area of specialisation, individuals do not show interest even in matters affecting their health.

The respondents were knowledgeable on the common signs/symptoms of malaria. Fever, weakness, headache, body pains, vomiting and loss of appetite were the common features recognised, similar to earlier reports by Fawole and Onadeko ${ }^{8}$ in Ibadan. Early recognition of symptomatology of malaria enhances early commencement of treatment. Although 57 (47.5\%) of the respondents commenced treatment on diagnosis of signs/symptoms of malaria, 63 (52.5\%) delayed for one to three days before initiating treatment. The delay in commencement of treatment after recognition of symptomatology of malaria corresponds with the findings of Hamel et $\mathrm{al}^{10}$ in Kenya, where majority delayed treatment till after two days.

Delay in treatment after diagnosis of signs/ symptoms of the disease could lead to complication and even death. There is a health facility on campus that provides health care services for staff and students. Delay in treatment in this community was probably due to the fact that they commenced treatment with antipyretics and analgesics stocked at home, which only catered for the symptoms, leaving the cause. ${ }^{11}$ This practice is encouraged by frequent television and radio adverts of such drugs as bosca (aspirin), etc, which patients are expected to take for three days before seeking help if symptoms do not subside. Meanwhile, the parasite multiplies in the system. Therefore, advert of drugs on mass media could be dangerous to public health if proper indications are not stated in the adverts.

Previous reports from Uganda, ${ }^{12}$ Congo $^{13}$ and Nigeria $^{8}$ revealed that orthodox medication was preferred for home management of malaria as, observed in this study. This is expected, as majority of the respondents believed that orthodox drugs are more effective, safer and a product of scientific research. The use of a combination of both orthodox and herbal preparations observed in this survey had been similarly reported by Ahorlu et $\mathrm{al}^{14}$ in Ghana. The use of this combination could have dangerous consequences arising from the complex reactions between the orthodox and herbal preparations. The use of herbal preparation for the treatment of malaria in this community is consistent with what obtains in other communities in Nigeria. Agbo is a common herbal preparation used in the treatment of malaria. Although most users have found it effective, the proportion of active ingredients in the preparations remains unknown. Users of the herbal preparations for treatment of malaria are those who claim that they do not visit hospitals because of the bureaucratic delays and the opinion that herbal medicines are readily available and cheap. The bureaucratic bottlenecks in most government hospitals in Nigeria have affected the health-seeking behaviour of some patients and caused dissatisfaction ${ }^{15}$ in hospital services. Others claimed that fake orthodox drugs are rampant in the market. The National Agency for Food and Drug Administration and Control (NAFDAC) ${ }^{16}$ has confirmed the presence of fake and adulterated drugs in the market and its potential harm to consumers. We hope that the war against fake drugs will eliminate the scourge.

In spite of the preference for orthodox drugs, the use of incorrect dosage still persists in the community as reported in Mali, ${ }^{17}$ Tanzania, ${ }^{3}$ Kenya $^{18}$ and Nigeria. ${ }^{8}$ The incorrect use of antimalarials such as chloroquine due to its bitter taste and associated allergic reactions have produced chloroquine-resistant strains of Plasmodium falciparum, leading to the development of 
new drugs that are also being abused. The persistent incorrect administration of common antimalarials continues to pose a huge problem in the efforts towards effective control of malaria in Nigeria and elsewhere in the world. ${ }^{17,18}$

The most widely used preventive method against malaria was door/window nettings. These may not be very effective, as weathering of the gauze causes breakage, thus allowing the passage of Anopheles vectors. Interestingly, some of the respondents slept under untreated bed nets to avoid mosquito bites. Those who found it convenient to sleep under untreated bed nets would comfortably adopt insecticide treated bed nets (ITN). However, this effective and cheap ${ }^{19}$ method of prevention is not available in Edo State, Nigeria.

Home management of malaria is an important strategy that needs to be sustained. Household members need to be educated on the importance of commencing treatment early. However, inadequate treatment regimen remains a hindrance to the success of this vital strategy. There is need to educate all strata of the society on the correct treatment regimen of common antimalarials to reduce the impact of the disease. This study has shown that no strata of the society should be assumed to have been adequately informed on health matters. Health education, information and communication should be made available to all.

\section{References}

1. WHO. Malaria - a global crisis. Quart Bull WHO 2000; 15(2).

2. WHO. Africa Malaria Day: Message of the Regional Director, 24th April 2002.

3. WHO. Roll-Back Malaria. RBM Advocacy Guide. WHO /CDS/ RBM/2000. 26.

4. WHO. TDR in WHO. A time for change rolling back malaria. TDR Reports. No. 58, 1999.
5. Umeh RE. Home management of malaria. Quart Bull WHO 2000; 15(2).

6. National Population Commission. Nigerian Demographic and Health Survey, 1999. Carvelton: NPC and ORC/Macro/2000.

7. Federal Ministry of Education, Office of the Honourable Minister. Circular letter to all vice-chancellors, federal universities, director of inter-university centres. Ref. HME/FME/332/vol. 1/33. 25th Jan. 2002.

8. Fawole OI and Onadeko MO. Knowledge and home management of malaria fever by mothers and caregivers of under-five children. W Afr J Med 2001; 20(2): 152-157.

9. Martin TC. Women's education and fertility studies. Fam Plann 1995; 26: 187-202.

10. Harmel MJ, Odhacha A, Roberts JM and Deming MS. Malaria control in Bungoma District, Kenya: a survey of home treatment of children with fever, bed net use and attendance of antenatal clinics. Bull WHO 2001; 79(11): 1014-23.

11. Tarimo DS, Lwihula GK, Minjhas JN and Bygbjera IC. Mother's perception and knowledge on childhood malaria in the holoendemic Kibaha District, Tanzania. Implication for malaria control and IMCI strategy. Trop Med Intern Health 2000; 5(3): 179-184.

12. Lubanga RG, Norman S, Ewban KD and Karamagi C. Maternal diagnosis and treatment of children's fever in endemic malaria zone of Uganda. Implications for the malaria control programme. Acta Trop 1997; 68(1): 53-64.

13. Talani P, Samba G and Moyem G. Management of child fever in the battle against malaria in Congo Brazzaville. Bull Soc Pathol Exot 2002; 95(1): 47-49.

14. Ahorlu CK, Dunyo SK, Afari EA, Koram KA and Nkrumah FK. Malaria related beliefs and behaviours in southern Ghana: implications for treatment, prevention and control. Trop Med Int Health 1997; 2(5): 488499.

15. Rusmiyati R. Focus Group for Developing a Patient Satisfaction Questionnaire. Directorate of Health Centre Development, Ministry of Health, Jarkata, Indonesia, 1997. 
16. Odutola A. Afro-nets. Fake drugs, weapon of terrorism - says Nigeria drug control czar. National Agency for Food and Drug Administration and Control (NAFDAC), 2003.

17. Thera MA, D'Alessandro CL, Thiero M, Ouedraogo A, Packoru J, Souleymane OA, Fane M, Ade G, Alvez F and Doumbo O. Child Malaria treatment practices among mothers in the district of Yanfolila, Sikasso Region, Mali. Trop Med Int Health 2000;
5(12): 876-881.

18. Ruebush TK, Kern MK, Campbell CC and Oloo AJ. Self-treatment of malaria in a rural area of western Kenya. Bull WHO 1995; 73(2): 229-236.

19. Curtis CF. Impregnated bednets; malaria control and child mortality in Africa. Trop Med Int Health 1996; 1(2): 137-138. 